\title{
Simulation of associative learning with the replaced elements model
}

\author{
STEVen Glautier \\ University of Southampton, Southampton, England
}

\begin{abstract}
Associative learning theories can be categorized according to whether they treat the representation of stimulus compounds in an elemental or a configural manner. Since it is clear that a simple elemental approach to stimulus representation is inadequate, there have been several attempts to produce more elaborate elemental models. One recent approach, the replaced elements model (Wagner, 2003), reproduces many results that have until recently been uniquely predicted by Pearce's configural theory (Pearce, 1994). Although it is possible to simulate the replaced elements model using "standard" simulation programs, the generation of the correct stimulus representation is complex. The present article describes a method for simulation of the replaced elements model and presents the results of two example simulations that show differential predictions of replaced elements and Pearce's configural theory.
\end{abstract}

A simple application of the Rescorla-Wagner model (RWM) of associative learning treats stimulus inputs as simple experimenter-defined elements (Rescorla \& Wagner, 1972). For example, in Mackintosh's 1976 study of overshadowing in rats, a compound of two stimuli, $\mathrm{A}$ and $\mathrm{B}$, was trained as a signal for electric shock (Mackintosh, 1976). In this procedure, according to the RWM, the associative strength of A and B would increase on each trial, as specified in Equation 1:

$$
\Delta \mathrm{V}_{n}=\alpha \beta\left[\left(\lambda-\left(\mathrm{VA}_{n-1}+\mathrm{VB}_{n-1}\right)\right] .\right.
$$

Equation 1 states that the change in associative strength for each of $\mathrm{A}$ and $\mathrm{B}\left(\Delta \mathrm{V}_{n}\right)$ is a function of two learning rate parameters $(\alpha$ and $\beta)$ and the difference between an asymptote $(\lambda)$ and the sum of the strengths of $A$ and $B$ on the previous trial $\left(\mathrm{VA}_{n-1}\right.$ and $\left.\mathrm{VB}_{n-1}\right)$. In this model, after a number of trials, it would be expected that VA and VB would both approach $1 / 2 \lambda$ if $A$ and $B$ are of equal salience. In contrast, if A was trained alone, its asymptotic associative strength would be $\lambda$. Unfortunately, this does not lead to the prediction that the response to $\mathrm{A}$ after training with an $\mathrm{AB}$ compound will be half of the magnitude of that seen to A after training with A alone. This prediction does not follow for at least two reasons. First, there is no accepted mapping between associative strength and response strength, and second, there is no accepted account of the process by which associative mechanisms treat stimulus compounds.

The assumption that stimulus compounds are treated by the associative mechanism simply as experimenterdefined elements has been challenged on a number of grounds. For example, there are numerous experiments that show that both animals and humans can learn to re- spond more to the elements than to the compound in negative patterning discriminations (e.g., Lachnit \& Kimmel, 2000; Woodbury, 1943). In negative patterning, elements are reinforced, whereas a compound of those elements is nonreinforced (i.e., $\mathrm{A}+, \mathrm{B}+$, and $\mathrm{AB}-$ trials, where "+" and "-" symbolize reinforcement and nonreinforcement, respectively). This type of discrimination cannot be solved by treating the stimulus inputs as simple elements, but, under the assumption that compounding produces a unique configural cue (Wagner \& Rescorla, 1972), the RWM can solve this type of problem. For instance, the experimenter may present a light (A) and a tone (B), but the organism represents the light-tone compound as ABX, where $\mathrm{X}$ is the unique configural cue that occurs in the presence of the $\mathrm{AB}$ compound. Apart from the fact that negative patterning discriminations can be learned, some experimental evidence has been provided that a unique cue is actually generated when two stimuli are compounded (e.g., Rescorla, 1973).

Negative patterning discriminations have been dealt with in alternatives to the unique-cue extension of the RWM. In one approach, a configural theory (CT) has been proposed (Pearce, 1987, 1994). In Pearce's CT, changes in associative strength are calculated in a similar way to the computations in the RWM. But CT assumes that entire stimulus patterns gain associative strength. Thus, in negative patterning, three distinct patterns would undergo associative changes: a representation of $\mathrm{A}$, a representation of $\mathrm{B}$, and a representation of $\mathrm{AB}$. Because the $\mathrm{AB}$ compound has its own distinct representation and associative strength, the model is readily able to predict that negative patterning discriminations can be learned. Furthermore, CT has the advantage over the unique-cue ver- 
sion of the RWM because the unique-cue RWM predicts summation effects, despite the fact that summation does not always occur. For example, Aydin and Pearce have reported that responding to an $\mathrm{AB}$ compound did not exceed responding to the previously reinforced $\mathrm{A}$ and $\mathrm{B}$ elements (Aydin \& Pearce, 1995, 1997). The RWM would predict that after training $\mathrm{A}$ and $\mathrm{B}$, the associative strengths of $\mathrm{A}$ and $\mathrm{B}$ would summate in an $\mathrm{AB}$ compound, and hence, the response to $\mathrm{AB}$ should exceed that seen to either $\mathrm{A}$ or $B$ alone.

In what follows, a recent model for the processing of stimulus compounds, the replaced elements model (REM) will be described (Brandon, Vogel, \& Wagner, 2000; Wagner, 2003; Wagner \& Brandon, 2001). Although the computation of associative strengths in the REM is a straightforward application of the delta rule used in the RWM (Rescorla \& Wagner, 1972; Sutton \& Barto, 1981), the model is complex in its specification of the elements for which associative strengths need to be calculated. Nevertheless, it is of particular interest to evaluate the REM, because, although it is an "elemental model" of associative learning, it can behave either "configurally" or elementally as its key parameter, $R$, varies. As a result, the model is a challenge both to alternative elemental (e.g., RWM and its unique-cue variants) and configural (Pearce, $1987,1994)$ accounts of associative learning, which incorporate different mechanisms for processing of stimulus compounds. The present article outlines the key features of the REM and describes a method for specifying the REM elements so that REM predictions can be generated computationally. In addition, example simulations are presented. These show differential predictions of the REM and Pearce's configural theory.

In essence, the REM extends the idea that unique configural cues can be produced whenever two or more stimuli are compounded. The suggestion is that representation of a stimulus is composed of three types of elements: (1) elements that represent the stimulus independently of the context set by other stimuli, (2) elements that represent the stimulus in a context that encodes the presence of other stimuli, and (3) elements that represent the stimulus in a context that encodes the absence of other stimuli. For example, in order to represent a stimulus world consisting of two stimulus components A and B, the REM specifies elements of $\mathrm{A}$ that are always active when $\mathrm{A}$ occurs ( $\mathrm{Ai}$, read "A independent" elements), elements of A that represent $A$ in the presence of $B(A B$, read " $A$ in the presence of $B$ " elements), and elements of $A$ that represent $A$ in absence of B (Ab, read "A in the absence of B" elements). Representation of B is similarly specified by Bi, BA, and $\mathrm{Ba}$ elements. The parameter $R(0 \leq R \leq 1)$ dictates the proportion of stimulus elements that encode the context set by the presence and absence of other stimuli, and variation in $R$ renders the REM capable of behaving either elementally or configurally. For instance, when $R=0$, the entire representation of $A$ is made up of Ai elementsthere is no encoding of the presence or absence of other stimuli. When $R=.5$, half of the representation of $\mathrm{A}$ is made up of Ai elements and the other half encodes the presence/absence of other stimuli. This scheme will be expanded on in the description of the simulation program below (for further details, see Wagner, 2003).

For simple simulations of the REM, it is possible to adapt conventional programs for RWM simulations by choosing stimuli to represent the REM elements, providing that the numbers of stimuli representing the elements are proportional to those required in REM. For example, to simulate a world with two components A and B, with an $R$-value of .5, the A component would be represented by three elements, one to code $\mathrm{A}$, one to code $\mathrm{Ab}$, and one to code $\mathrm{AB}$, and the $\mathrm{B}$ component would be represented likewise, one for element $\mathrm{Bi}$, one for $\mathrm{Ba}$, and one to code $\mathrm{BA}$. The proportional representations required for $\mathrm{A}, \mathrm{B}$, and $\mathrm{AB}$ trials with $R=.5$ are illustrated in the left-hand side of Table 1, which shows how the conditioned stimulus (CS) inputs to a conventional RWM simulation of this scenario could be presented in a binary vector with length 6 . Referring to Table 1, conditioning on a trial involving CS A alone would require recalculation of $\mathrm{Ai}$ and $\mathrm{Ab}$ elements, whereas a trial with an $\mathrm{AB}$ compound would require computations on $\mathrm{Ai}, \mathrm{AB}, \mathrm{Bi}$, and $\mathrm{BA}$ elements.

Simulations of this two-component world are also possible for other values of $R$. The right-hand side of Table 1 shows proportional representations required for an $R$-value of .25. These proportions can be represented using a binary vector of length 10 for the CS encoding, three elements for each of $\mathrm{Ai}$ and $\mathrm{Bi}$, and one element for each of $\mathrm{Ab}, \mathrm{AB}, \mathrm{Ba}$, and $\mathrm{BA}$. On a trial with $\mathrm{CS} \mathrm{A}$ alone, all three Ai elements would need their associative strengths adjusted, along with the single Ab element. Once the correct proportional representations of different elements for each conditioning trial are established, the simulation can then proceed keeping track of the associative strengths of elements in the conventional way. Equation 1 shows how this works on conditioning trials involving A alone.

$$
\Delta \mathrm{V}=\alpha \beta\left\{\lambda-\left[\left(\sum_{k=1}^{3} \mathrm{VAi}\right)+\mathrm{VAb}\right]\right\}
$$

In Equation 2, the summation term indicates that there are three elements representing $\mathrm{Ai}$ (for a two-component model with $R=.25$ ) and one element representing $\mathrm{Ab}$, each of which needs to be included in the error term. Application to trials involving $\mathrm{B}$, and $\mathrm{AB}$ compounds, is ap-

Table 1

An Illustration of the Vector Inputs Required for an RWMBased Simulation of REM for $R$-Values of .5 and .25 to Represent All Combinations of Two Component Stimuli, A and B

\begin{tabular}{|c|c|c|c|c|c|c|c|}
\hline \multirow{3}{*}{$\begin{array}{l}\text { Input } \\
\text { Elements }\end{array}$} & \multicolumn{6}{|c|}{ CSs } & \multirow{3}{*}{$\begin{array}{l}\text { REM Element } \\
\text { Weight }(\omega)\end{array}$} \\
\hline & \multicolumn{3}{|c|}{$R=.5$} & \multicolumn{3}{|c|}{$R=.25$} & \\
\hline & $\mathrm{A}$ & $\mathrm{B}$ & $\mathrm{AB}$ & $\mathrm{A}$ & $\mathrm{B}$ & $\mathrm{AB}$ & \\
\hline $\mathrm{Ai}$ & 1 & 0 & 1 & 3 & 0 & 3 & $1-R$ \\
\hline $\mathrm{Ab}$ & 1 & 0 & 0 & 1 & 0 & 0 & $R$ \\
\hline $\mathrm{AB}$ & 0 & 0 & 1 & 0 & 0 & 1 & $R$ \\
\hline $\mathrm{Bi}$ & 0 & 1 & 1 & 0 & 3 & 3 & $1-R$ \\
\hline $\mathrm{Ba}$ & 0 & 1 & 0 & 0 & 1 & 0 & $R$ \\
\hline BA & 0 & 0 & 1 & 0 & 0 & 1 & $R$ \\
\hline
\end{tabular}

Note-With $R=.25$, three elements are required to represent each of $\mathrm{Ai}$ and $\mathrm{Bi}$. The far right-hand column shows REM weights for each element. 
Table 2

RWM Simulation of REM $(R=.25)$ Based on Equation 2 for Three Reinforced Trials

\begin{tabular}{lccccccccccc}
\hline Trial & $\lambda-\Sigma V$ & $\mathrm{Ai}_{1}$ & $\mathrm{Ai}_{2}$ & $\mathrm{Ai}_{3}$ & $\mathrm{Ab}$ & $\mathrm{AB}$ & $\mathrm{Bi}_{1}$ & $\mathrm{Bi}_{2}$ & $\mathrm{Bi}_{3}$ & $\mathrm{Ba}$ & $\mathrm{BA}$ \\
\hline $\mathrm{A}+$ & 1.0000 & .1250 & .1250 & .1250 & .1250 & .0000 & .0000 & .0000 & .0000 & .0000 & .0000 \\
$\mathrm{AB}+$ & .6250 & .2031 & .2031 & .2031 & .1250 & .0781 & .0781 & .0781 & .0781 & .0000 & .0781 \\
$\mathrm{~B}+$ & .7656 & .2031 & .2031 & .2031 & .1250 & .0781 & .1738 & .1738 & .1738 & .0957 & .0781 \\
\hline
\end{tabular}

Note-The column headed $\lambda-\Sigma V$ shows the error term at the start of each trial, and the numbers in the body of the table are associative strengths for each element after the trial.

proached in a similar way. Tests on A, B, and AB would then be carried out by adding the associative strengths of their respective elements; for example, for a test on A alone, add the associative strengths of all elements that represent $\mathrm{A}$ alone (i.e., three $\mathrm{Ai}$ and one $\mathrm{Ab}$ element).

Table 2 gives the results of an RWM simulation of REM for a sequence of three reinforced trials involving $\mathrm{A}, \mathrm{AB}$, and then $\mathrm{B}$, with $R=.25$ based on Equation 2 with $\alpha \beta=$ .125 . For more complex simulations involving multiple stimuli, for example, a three-stimulus world of A, B, and $\mathrm{C}$, or four stimuli, A-D and arbitrary $R$-values, the total number of stimulus elements required to represent the required proportions becomes very large, and construction of the vectors for different stimulus patterns is complex. As a result, a more effective method is suggested below.

\section{Method}

The alternative approach involves using single elements to represent each REM element and weighting the updates on the associative strengths of those elements to reflect their proportional representations in REM. Equation 3 illustrates the use of these weights in simulation of trials involving A alone.

$$
\Delta \mathrm{V}_{n}=\omega \alpha \beta[\lambda-(\mathrm{VAi}+\mathrm{VAb})]
$$

In the case of $R=.25$ for the two-component simulation, the weight $(\omega)$ for $\triangle \mathrm{VAi}$ is $(1-R)$, whereas $\omega$ for $\triangle \mathrm{VAb}$ is $R$. The far right-hand column of Table 1 shows weights required for all the elements involved in an REM simulation involving two stimuli. Table 3 shows the results of the same simulation carried out previously (in Table 2) but this time using weighted single elements to code for each component according to Equation 3. Inspection of Tables 2 and 3 shows that the associative strength of the single Ai component in Table 3 is the sum of the three individual Ai components from Table $2 .{ }^{1}$ Two further steps are required to facilitate simulations with arbitrary values of $R$ and arbitrary numbers of stimulus elements. The first is enumeration of the REM components present on a conditioning trial and the second is calculation of the correct weights for each component.

\section{Enumeration of REM Components}

Given a list of the stimuli that are present on a trial, and a list of stimuli that are absent, the algorithm REMElements (StimuliPresent, StimuliAbsent) returns the list of REM elements that are assumed to be active and whose associative strengths will need updating on that trial (see the Appendix and the text below for details of this algorithm). Returning to the two-component world involving
$\mathrm{A}$ and $\mathrm{B}$, the REM element list for a trial involving $\mathrm{A}$ alone would be returned from a function call of the form REMElements $(\mathrm{A}, \mathrm{b})$. The first parameter of this function call takes a list of the components that are actually present (in uppercase) and the second parameter takes a list of elements that are absent (in lowercase). If a threecomponent world (components A-C) was simulated, then a list of REM elements for a trial involving $\mathrm{BC}$ would be generated by the call REMElements (BC, a). The outputs from this algorithm for different stimulus combinations for a three-component simulation are given in Table 4. The left-hand side of Table 4 lists all of the REM elements that could be active in the three-component world. The main body of the table contains $0 \mathrm{~s}$ and $1 \mathrm{~s}$ to indicate whether an REM element is active, given the presence of the stimulus components listed across the top of the table. For example, if $\mathrm{AB}$ was presented, then REM elements $\mathrm{Ai}, \mathrm{AB}, \mathrm{Ac}, \mathrm{ABc}, \mathrm{Bi}, \mathrm{BA}, \mathrm{Bc}, \mathrm{BAc}$ would be activated and the call REMElements (AB, c) would generate the list of strings $\mathrm{A}, \mathrm{AB}, \mathrm{Ac}, \mathrm{ABc}, \mathrm{B}, \mathrm{BA}, \mathrm{Bc}, \mathrm{BAc}$ corresponding to those elements. Note that $\mathrm{A}, \mathrm{B}$, and $\mathrm{C}$ correspond to $\mathrm{Ai}, \mathrm{Bi}$, and $\mathrm{Ci}$ - the suffix $\mathrm{i}$, used in the text, is dropped as redundant.

The Appendix gives further details of the algorithm that generates a list of all the REM elements present on a trial for an arbitrary number of components. This algorithm can be incorporated into a simulation program using two lists, TList and SList. A new TList is created on each trial of the simulation and uses the REMElements function to establish which elements are present on that trial. SList is created once for each simulation and is a list of all the elements that have already been encountered during the simulation, along with their current associative strengths. On any trial, the first step is to populate TList with the elements that are present on that trial. Next, referring to SList, update the associative strengths of all the elements in TList. If there is an element in TList but not in SList, then it has never been encountered before and its strength can be set to a default (e.g., 0). Next, the change in as-

Table 3

Simulation of REM Based on Equation 3 for Three Reinforced Trials

\begin{tabular}{lccccccc}
\hline Trial & $\lambda-\Sigma V$ & $\mathrm{Ai}$ & $\mathrm{Ab}$ & $\mathrm{AB}$ & $\mathrm{Bi}$ & $\mathrm{Ba}$ & $\mathrm{BA}$ \\
\hline $\mathrm{A}+$ & 1.0000 & .3750 & .1250 & .0000 & .0000 & .0000 & .0000 \\
$\mathrm{AB}+$ & .6250 & .6094 & .1250 & .0781 & .2344 & .0000 & .0781 \\
$\mathrm{~B}+$ & .7656 & .6094 & .1250 & .0781 & .5215 & .0957 & .0781 \\
\hline
\end{tabular}

Note-The column headed $\lambda-\Sigma V$ shows the error term at the start of each trial, and the numbers in the body of the table are associative strengths for each element after the trial. 
Table 4

Illustration of REM Element Strings Required to Represent All Combinations of CSs in a Three-Component World Involving A, B, and C

\begin{tabular}{|c|c|c|c|c|c|c|c|c|}
\hline \multirow{2}{*}{$\begin{array}{c}\text { REM } \\
\text { Element } \\
\text { String }\end{array}$} & \multicolumn{7}{|c|}{ Stimulus Components } & \multirow{2}{*}{$\begin{array}{c}\text { REM } \\
\text { Element } \\
\text { Weight }(\omega)\end{array}$} \\
\hline & $\mathrm{A}$ & B & $\mathrm{C}$ & $\mathrm{AB}$ & $\mathrm{AC}$ & $\mathrm{BC}$ & $\overline{A B C}$ & \\
\hline $\mathrm{Ai}$ & 1 & 0 & 0 & 1 & 1 & 0 & 1 & $(1-R)^{2}$ \\
\hline $\mathrm{AB}$ & 0 & 0 & 0 & 1 & 0 & 0 & 1 & $R *(1-R)$ \\
\hline $\mathrm{Ab}$ & 1 & 0 & 0 & 0 & 1 & 0 & 0 & $R *(1-R)$ \\
\hline $\mathrm{AC}$ & 0 & 0 & 0 & 0 & 1 & 0 & 1 & $R *(1-R)$ \\
\hline $\mathrm{Ac}$ & 1 & 0 & 0 & 1 & 0 & 0 & 0 & $R *(1-R)$ \\
\hline $\mathrm{ABC}$ & 0 & 0 & 0 & 0 & 0 & 0 & 1 & $R^{2}$ \\
\hline $\mathrm{ABc}$ & 0 & 0 & 0 & 1 & 0 & 0 & 0 & $R^{2}$ \\
\hline $\mathrm{ACb}$ & 0 & 0 & 0 & 0 & 1 & 0 & 0 & $R^{2}$ \\
\hline $\mathrm{Abc}$ & 1 & 0 & 0 & 0 & 0 & 0 & 0 & $R^{2}$ \\
\hline $\mathrm{Bi}$ & 0 & 1 & 0 & 1 & 0 & 1 & 1 & $(1-R)^{2}$ \\
\hline $\mathrm{BA}$ & 0 & 0 & 0 & 1 & 0 & 0 & 1 & $R *(1-R)$ \\
\hline $\mathrm{Ba}$ & 0 & 1 & 0 & 0 & 0 & 1 & 0 & $R *(1-R)$ \\
\hline $\mathrm{BC}$ & 0 & 0 & 0 & 0 & 0 & 1 & 1 & $R *(1-R)$ \\
\hline $\mathrm{Bc}$ & 0 & 1 & 0 & 1 & 0 & 0 & 0 & $R *(1-R)$ \\
\hline $\mathrm{BAC}$ & 0 & 0 & 0 & 0 & 0 & 0 & 1 & $R^{2}$ \\
\hline BAc & 0 & 0 & 0 & 1 & 0 & 0 & 0 & $R^{2}$ \\
\hline $\mathrm{BCa}$ & 0 & 0 & 0 & 0 & 0 & 1 & 0 & $R^{2}$ \\
\hline $\mathrm{Bac}$ & 0 & 1 & 0 & 0 & 0 & 0 & 0 & $R^{2}$ \\
\hline $\mathrm{Ci}$ & 0 & 0 & 1 & 0 & 1 & 1 & 1 & $(1-R)^{2}$ \\
\hline $\mathrm{CA}$ & 0 & 0 & 0 & 0 & 1 & 0 & 1 & $R *(1-R)$ \\
\hline $\mathrm{Ca}$ & 0 & 0 & 1 & 0 & 0 & 1 & 0 & $R *(1-R)$ \\
\hline $\mathrm{CB}$ & 0 & 0 & 0 & 0 & 0 & 1 & 1 & $R *(1-R)$ \\
\hline $\mathrm{Cb}$ & 0 & 0 & 1 & 0 & 1 & 0 & 0 & $R *(1-R)$ \\
\hline $\mathrm{CAB}$ & 0 & 0 & 0 & 0 & 0 & 0 & 1 & $R^{2}$ \\
\hline $\mathrm{CAb}$ & 0 & 0 & 0 & 0 & 1 & 0 & 0 & $R^{2}$ \\
\hline $\mathrm{CBa}$ & 0 & 0 & 0 & 0 & 0 & 1 & 0 & $R^{2}$ \\
\hline $\mathrm{Cab}$ & 0 & 0 & 0 & 0 & 0 & 0 & 0 & $R^{2}$ \\
\hline$\Sigma$ weights & 1 & 1 & 1 & 2 & 2 & 2 & 3 & \\
\hline
\end{tabular}

Note-The weights $(\omega)$ required in Equation 3 for each element are shown in the right-hand column, and the sum of the weights for each trial is given in the bottom row. For the purpose of weight determination, the length of the REM element string does not include the "i"- $\mathrm{Ai}, \mathrm{Bi}$, and $\mathrm{Ci}$ all have length 1 .

sociative strength for each element of TList is computed using Equation 4, and these change values are added to the associative strengths already recorded for each element in TList.

$$
\Delta \mathrm{V}_{k}=\omega_{k} \alpha \beta\left[\lambda-\left(\sum_{k=1}^{n} \mathrm{~V}_{k}\right)\right]
$$

In Equation 4, the summation in the error term (the major parenthesized term) is over the $n$ elements present in TList - that is, this is the sum of the associative strengths of all of the elements used in REM to represent the particular pattern of CSs present on that trial. The $\omega$ values for each element are required (see below), but once these changes have been calculated, these values are added to those already present in TList, and to complete the trial, SList is updated with new associative strengths for all of those stimuli that are in TList.

\section{Calculation of REM Component Weights}

A major feature of the present approach to simulation of REM is the use of a single element to represent each REM component instead of using multiple elements (see Table 1 and the introductory text). This simplification allows relatively straightforward simulations involving arbitrary numbers of stimuli and values of $R$, but requires calculation of weights ( $\omega$ values) for each REM component for use in Equation 4. Inspection of the right-hand columns of Tables 1 and 4 shows that the weights for components vary according to the overall "dimensionality" of the simulation. For example, in Table 1, where a twostimulus world of A and B is simulated, the weight for Ai is $1-R$. In Table 4, where a three-stimulus world is simulated, the weight for Ai is $(1-R)^{2}$. Different weights are required to ensure that the overall rate of conditioning reflects the number of experimenter-defined stimuli that are present in a trial. The bottom row of Table 4 shows that the sum of the weights for all of the REM components present on a trial is $N$, where $N$ is the number of CSs present. This also holds for the 2-D world of Table 1, where different REM components would be used to represent, for example, $\mathrm{AB}$. Thus, different $\omega$ values are required for REM components as the dimensionality of the simulation varies. The method for determining the $\omega$ values ensures that the results of a simulation will be the same whatever the dimensionality of the simulation; for example, a sequence of $\mathrm{A}+, \mathrm{AB}+, \mathrm{B}+$ would yield the same results whether conducted in a two-stimulus or a four-stimulus simulation. Calculation of the required weights can be achieved using the binomial expansion in Equation 5.

$$
(R+S)^{(D-1)} .
$$

Equation 5 is a general expression for REM weights, where $\mathrm{D}$ is the dimensionality of the simulation being conducted and $S=1-R$. In a simulation of a two-stimulus world, the elements $\mathrm{Ab}, \mathrm{AB}, \mathrm{Ba}$, and $\mathrm{BA}$ have a weight of $R$, whereas $\mathrm{Ai}$ and $\mathrm{Bi}$ have a weight of $(1-R)$. In simulation of a three-stimulus world, Equation 5 expands to

$$
R^{2}+2 R S+S^{2},
$$

and the weights $R^{2}, R(1-R)$, and $(1-R)^{2}$ are applied to REM element strings (generated as described in the Appendix) with lengths 3,2 , and 1, respectively. See Tables 1 ,

Table 5

REM Element Strings and Weights Generated From a Call on REM Elements (ABC, de)

\begin{tabular}{llll}
\hline A Elements & B Elements & C Elements & $\begin{array}{c}\text { Reight }(\omega) \\
\text { Element }\end{array}$ \\
\hline $\mathrm{A}$ & $\mathrm{B}$ & $\mathrm{C}$ & $(1-R)^{4}$ \\
$\mathrm{Ad}$ & $\mathrm{Bd}$ & $\mathrm{Cd}$ & $R *(1-R)^{3}$ \\
$\mathrm{Ae}$ & $\mathrm{Be}$ & $\mathrm{Ce}$ & $R *(1-R)^{3}$ \\
$\mathrm{Ade}$ & $\mathrm{Bde}$ & $\mathrm{Cde}$ & $R^{2} *(1-R)^{2}$ \\
$\mathrm{AB}$ & $\mathrm{BA}$ & $\mathrm{CA}$ & $R *(1-R)^{3}$ \\
$\mathrm{ABd}$ & $\mathrm{BAd}$ & $\mathrm{CAd}$ & $R^{2} *(1-R)^{2}$ \\
$\mathrm{ABe}$ & $\mathrm{BAe}$ & $\mathrm{CAe}$ & $R^{2} *(1-R)^{2}$ \\
$\mathrm{ABde}$ & $\mathrm{BAde}$ & $\mathrm{CAde}$ & $R^{3} *(1-R)$ \\
$\mathrm{AC}$ & $\mathrm{BC}$ & $\mathrm{CB}$ & $R *(1-R)^{3}$ \\
$\mathrm{ACe}$ & $\mathrm{BCd}$ & $\mathrm{CBd}$ & $R^{2} *(1-R)^{2}$ \\
$\mathrm{ACd}$ & $\mathrm{BCe}$ & $\mathrm{CBe}$ & $R^{2} *(1-R)^{2}$ \\
$\mathrm{ACde}$ & $\mathrm{BCde}$ & $\mathrm{CBde}$ & $R^{3} *(1-R)$ \\
$\mathrm{ABC}$ & $\mathrm{BAC}$ & $\mathrm{CAB}$ & $R^{2} *(1-R)^{2}$ \\
$\mathrm{ABCe}$ & $\mathrm{BACd}$ & $\mathrm{CABd}$ & $R^{3} *(1-R)$ \\
$\mathrm{ABCd}$ & $\mathrm{BACd}$ & $\mathrm{CABe}$ & $R^{3} *(1-R)$ \\
$\mathrm{ABCde}$ & $\mathrm{BACde}$ & $\mathrm{CABde}$ & $R^{4}$ \\
\hline
\end{tabular}


Table 6

REM Element Weights $(\omega)$ According to REM Element String Lengths and Dimensionality of Simulation

\begin{tabular}{|c|c|c|c|c|c|}
\hline \multirow{2}{*}{$\begin{array}{c}\text { REM Element } \\
\text { String Length } \\
\text { (Example) }\end{array}$} & \multicolumn{5}{|c|}{ Dimensionality } \\
\hline & $2-\mathrm{D}$ & $3-\mathrm{D}$ & 4-D & $5-\mathrm{D}$ & $6-\mathrm{D}$ \\
\hline $1(\mathrm{~A})$ & $1-R$ & $(1-R)^{2}$ & $(1-R)^{3}$ & $(1-R)^{4}$ & $(1-R)^{5}$ \\
\hline $2(\mathrm{Ba})$ & $R$ & $R *(1-R)$ & $R *(1-R)^{2}$ & $R *(1-R)^{3}$ & $R *(1-R)^{4}$ \\
\hline $3(\mathrm{CAb})$ & - & $R^{2}$ & $R^{2} *(1-R)$ & $R^{2} *(1-R)^{2}$ & $R^{2} *(1-R)^{3}$ \\
\hline $4(\mathrm{DBac})$ & - & - & $R^{3}$ & $R^{3} *(1-R)$ & $R^{3} *(1-R)^{2}$ \\
\hline 5 (ABCde) & - & - & - & $R^{4}$ & $R^{4} *(1-R)$ \\
\hline 6 (FAbcde) & - & - & - & - & $R^{5}$ \\
\hline
\end{tabular}

4 , and 5 for examples of weights generated for elements from 2-, 3-, and 5-D simulations. Table 6 lists all weights needed for models involving up to 6-D simulations.

\section{Discussion and Example Simulations}

Although simulations of REM can be carried out using conventional RWM approaches, generation of the required elements is cumbersome; however, the foregoing illustrates an alternative method for simulation of the REM. The method includes algorithmic generation of REM stimulus components from a list of experimenter-defined stimuli so that, subject to computing limitations, simulations can be carried out with arbitrary numbers of stimuli and values of $R$. Simulation of REM is of interest because it represents an attempt to overcome limitations of purely elemental approaches such as the RWM. For example, the RWM cannot generate appropriate predictions for nonlinear discriminations such as negative patterning. Although it is possible for the RWM to solve this type of discrimination using unique cues to code stimulus compounds, the unique-cue approach still predicts summation when summation effects do not always occur (Aydin \& Pearce, 1995, 1997).

Both Pearce's configural model (Pearce, 1994) and the REM are alternatives to the RWM that can generate the correct predictions for the negative patterning design, and both indicate that summation may or may not occur. In the case of Pearce's model, summation is predicted when there are common elements to the trained stimuli. For example, a test on $\mathrm{ABC}$ will lead to summation after reinforced $\mathrm{AC}$ and $\mathrm{BC}$ trials. In the case of the REM, summation is predicted to occur with low $R$ values. What then differentiates these models? Wagner referred to several experiments in which the different predictions of the RWM and Pearce's configural model had both been supported, and showed how the REM could predict the outcomes subject to variation in $R$ (Wagner, 2003). Nonetheless, the most interesting tests of these theoretical models are from designs that make parameter-free differential predictions.

Two examples will be given. The first is a simulation of a feature-negative design $(\mathrm{A}+, \mathrm{AB}-)$, which is predicted to be made more difficult by $\mathrm{CT}$ after the addition of a common feature $(\mathrm{AC}+, \mathrm{ABC}-)$. On the other hand, the addition of the common feature is predicted to make the discrimination easier by the REM. Figure 1 shows the results of this simulation and the clear differential predictions made by $\mathrm{CT}$ and REM. In the limit $(R=1)$, the two discriminations are equivalent in the REM, since there is no overlap in the components for the reinforced and nonreinforced trials. The REM $(R<1)$ suggests that the common feature will facilitate the discrimination because inhibition develops more rapidly on the $\mathrm{ABC}$ trials and, as a result, excitation develops more rapidly on the $\mathrm{AC}$ trials. On the other hand, CT suggests that the addition of the common feature will increase the similarity of the
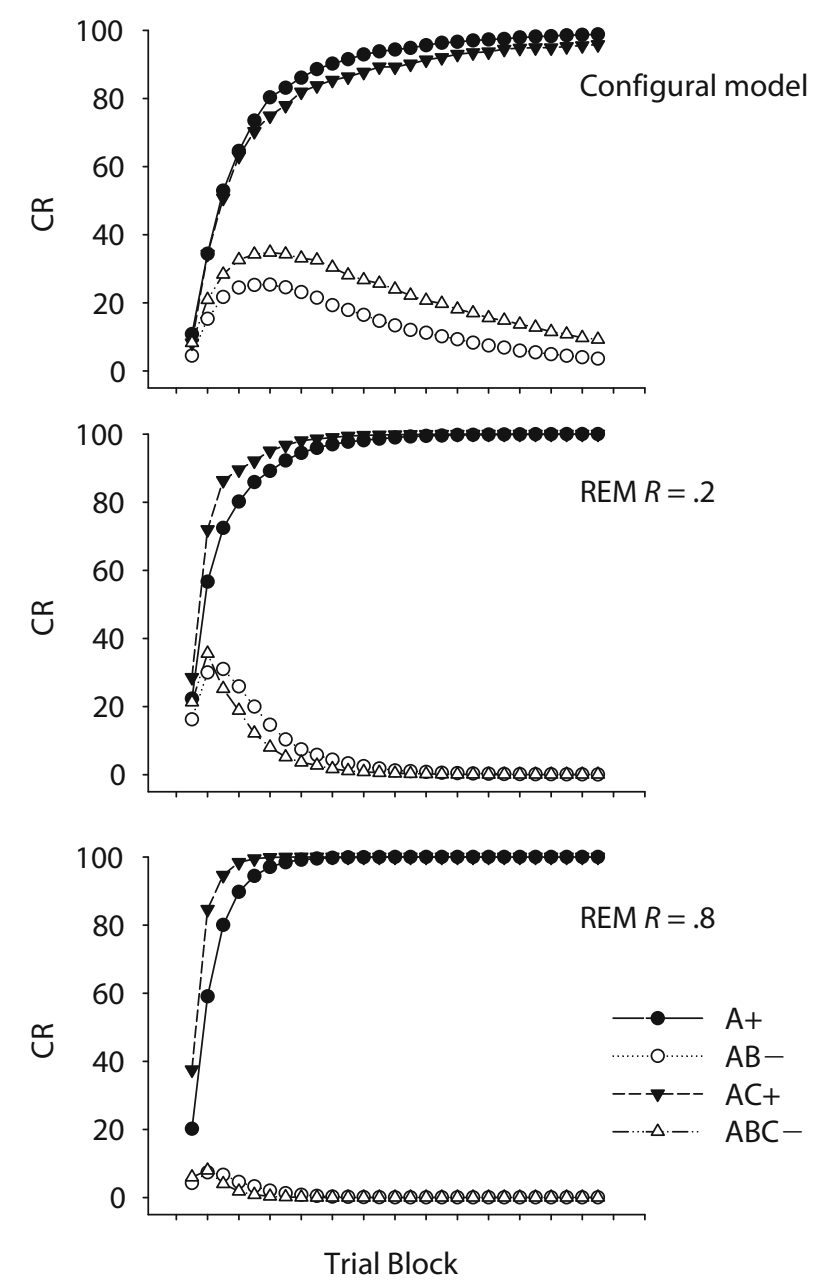

Figure 1. Simulations of feature-negative discriminations with $(\mathrm{AC}+, \mathrm{ABC}-)$ and without $(\mathrm{A}+, \mathrm{AB}-)$ common features. The predictions from Pearce's configural model and from the REM with $R=.2$ and $R=.8$ are shown. The abscissa shows associative strength $(V) \times 100$. See text for further details. 
stimuli that signal reinforced and nonreinforced trials and make the discrimination more difficult. Evidence has previously been obtained in support of the predictions of CT (e.g., Pearce \& Redhead, 1993). The second example is a contrast between a simple $(\mathrm{A}+, \mathrm{B}+, \mathrm{AB}-)$ and a complex $(\mathrm{AB}+, \mathrm{CD}+, \mathrm{ABCD}-)$ negative patterning discrimination (Figure 2). According to the predictions of $\mathrm{CT}$, there is equivalence in the similarity relations in the two discriminations, so the simple and complex designs should be equally difficult. In the REM though, the complex discrimination is predicted to be easier, because more stimuli are present on each trial, so both excitatory and inhibitory conditioning proceed more rapidly. When $R=1$, there is no overlap between the nonreinforced stimulus compounds, so the elements representing $\mathrm{AB}$ (simple) and ABCD (complex) do not become inhibitory and the discrimination only involves increases in excitatory strength for the reinforced stimuli $\mathrm{A} / \mathrm{B}$ (simple) and $\mathrm{AB} / \mathrm{CD}$ (complex). Although increasing the complexity of a negative
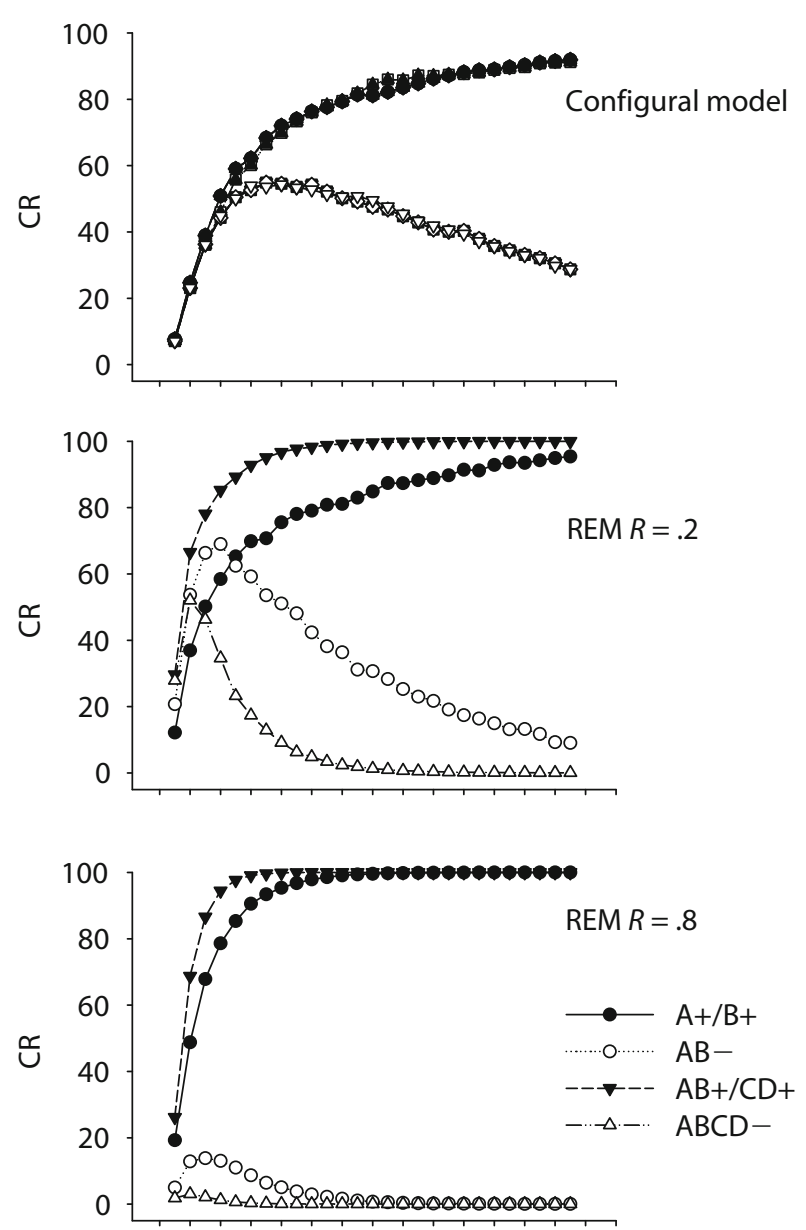

Trial Block

Figure 2. Simulations of simple $(A+/ B+/ A B-)$ and complex $(\mathrm{AB}+/ \mathrm{CD}+/ \mathrm{ABCD}-)$ negative patterning. The predictions from Pearce's configural model and from the REM with $R=.2$ and $R=.8$ are shown. The abscissa shows associative strength $(V) \times$ 100. See text for further details.
Table 7 Sample Simulation Data From Figures 1 and 2

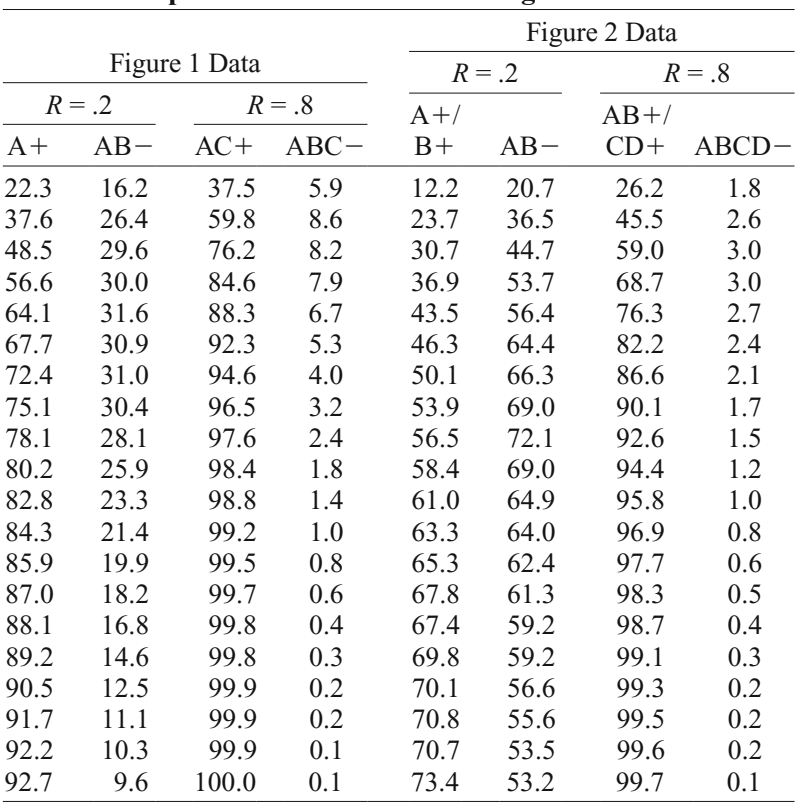

patterning design has been found to increase the difficulty of the discrimination, in support of $\mathrm{CT}$, this result has been found by increasing the similarity between the reinforced and nonreinforced cues (Pearce \& Redhead, 1993).

The simulations shown in Figures 1 and 2 were carried out as follows. The learning rate parameter $\alpha$ was set at .005 , whereas $\beta$ was set at 1 or .5 for reinforced and nonreinforced trials, respectively. For the simulations in Figure 1, 1,800 trials of each type were carried out in a randomized order. The simulation was stopped after every 45 trials, and tests were carried out. The values in the figure are averaged over 10 runs. The $\mathrm{A}+/ \mathrm{AB}-$ simulation was carried out separately from the $\mathrm{AC}+/ \mathrm{ABC}-$ simulation. The same procedure was followed for Figure 2 except that 1,200 trials of each type were carried out. The $\mathrm{A}+/ \mathrm{B}+/ \mathrm{AB}-$ simulation was run separately from the $\mathrm{AB}+/ \mathrm{CD}+/ \mathrm{ABCD}-$ simulation. Table 7 includes the data from Figures 1 and 2 for the first 20 sample points for selected trial types.

\section{AUTHOR NOTE}

I thank Alan Wagner for his helpful comments and elaborations on early attempts at simulation and Ed Redhead for discussion and comments on a draft of this article. Correspondence concerning this article should be addressed to S. Glautier, School of Psychology, University of Southampton, Southampton SO17 1BJ, England (e-mail: spg@soton.ac.uk).

\section{REFERENCES}

Aydin, A., \& Pearce, J. M. (1995). Summation in autoshaping with short and long duration stimuli. Quarterly Journal of Experimental Psychology, 42B, 215-234.

Aydin, A., \& Pearce, J. M. (1997). Some determinants of response summation. Animal Learning \& Behavior, 25, 108-121.

Brandon, S., Vogel, E. H., \& Wagner, A. R. (2000). A componential view of configural cues in generalization and discrimination in Pavlovian conditioning. Behavioural \& Brain Research, 110, 67-72.

LaChNit, H., \& Kimmel, H. D. (2000). Experimental manipulation of a unique cue in Pavlovian SCR conditioning with humans. Biological Psychology, 53, 105-129. 
Mackintosh, N. J. (1976). Overshadowing and stimulus intensity. Animal Learning \& Behavior, 4, 186-192.

Pearce, J. M. (1987). A model of stimulus generalisation for Pavlovian conditioning. Psychological Review, 94, 61-73.

Pearce, J. M. (1994). Similarity and discrimination: A selective review and a connectionist model. Psychological Review, 101, 587-607.

Pearce, J. M., \& Redhead, E. S. (1993). The influence of an irrelevant stimulus on two discriminations. Journal of Experimental Psychology: Animal Behavior Processes, 19, 180-190.

RESCORLA, R. A. (1973). Evidence for a "unique stimulus" account of configural conditioning. Journal of Comparative \& Physiological Psychology, 85, 331-338.

Rescorla, R. A., \& Wagner, A. R. (1972). A theory of Pavlovian conditioning: Variations in the effectiveness of reinforcement and non-reinforcement. In A. H. Black \& W. F. Prokasy (Eds.), Classical conditioning II: Current research and theory (pp. 64-69). New York: Appleton-Century-Crofts.

Sutton, R. S., \& Barto, A. G. (1981). Toward a modern theory of adaptive networks: Expectation and prediction. Psychological Review, 88, $135-170$.
Wagner, A. R. (2003). Context-sensitive elemental theory. Quarterly Journal of Experimental Psychology, 56B, 7-29.

WAGNER, A. R., \& BRANDON, S. E. (2001). A componential theory of Pavlovian conditioning. In R. R. Mowrer \& S. B. Klein (Eds.), Handbook of contemporary learning theories (pp. 23-64). Mahwah, NJ: Erlbaum.

WAGNer, A. R., \& Rescorla, R. A. (1972). Inhibition in Pavlovian conditioning: Application of a theory. In R. A. Boakes \& M. S. Halliday (Eds.), Inhibition and learning (pp. 301-340). London: Academic Press.

Woodbury, C. B. (1943). The learning of stimulus patterns by dogs. Journal of Comparative Psychology, 35, 29-40.

\section{NOTE}

1. In order to produce the same numerical values, the product of the learning rate parameters $(\alpha \beta)$ for the REM simulation was set at .5. Without this adjustment, the RWM simulation of REM would result in faster changes in associative strength as more stimuli are conditioned on each trial.

\section{APPENDIX}

The function REMElements (StimuliPresent, StimuliAbsent) takes two strings as its parameters, StimuliPresent and StimuliAbsent, and returns a list of strings. Each string on the returned list represents one of the REM elements that are present, given the parameters, and the returned list is a complete enumeration of those elements required by the REM to represent the CSs present on that trial. The StimuliPresent string is an enumeration of the CSs actually present; the StimuliAbsent string enumerates those CSs that are absent. For example, in simulation involving four CS components (A-D), a trial with A alone would require a call REMElements (A,bcd), whereas a trial of ACD would need the call REMElements (ACD, b). In a simulation involving five CS components (A-E), the same trials would be called with REMElements (A,bcde) and REMElements (ACD,be).

REMElements makes use of three lists. AList is generated from the StimuliAbsent string, whereas PList is generated from the StimuliPresent string. RList is the list that the function returns; it is generated by merging AList and PList. In pseudocode, the principal algorithm of REMElements is:

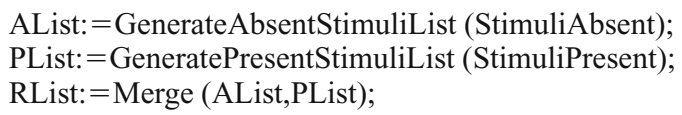

GenerateAbsentStimuliList operates by generating substrings of length 1..n, each of which is a unique combination of characters from the string StimuliAbsent, where $n$ is the length of StimuliAbsent. In pseudocode:

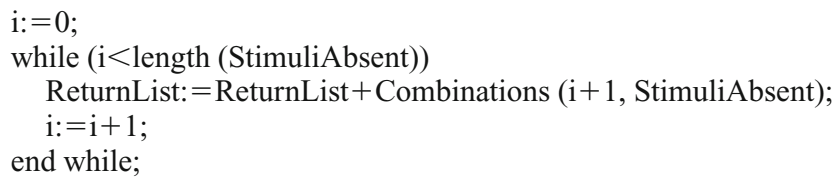

The call Combinations $(i+1$, StimuliAbsent) returns a list of all combinations of characters in StimuliAbsent with length $\mathrm{i}+1$. As examples, the call Combinations $(1, \mathrm{abc})$ would return a list containing elements $\mathrm{a}, \mathrm{b}$, and $\mathrm{c}$; Combinations $(2, a b c)$ would return elements $a b$, ac, and bc; whereas Combinations $(3, a b c)$ would return a list with a single element abc. As a result, the call GenerateAbsentStimuliList (abc) would produce the ReturnList consisting of elements a, b, c, ab, ac, bc, abc. The call GenerateAbsentStimuliList (de) would produce the ReturnList consisting of elements d, e, de.

GeneratePresentStimuliList operates on similar principles but produces calls on the Combinations function with substrings of StimuliPresent. Substrings of StimuliPresent are generated by rotating the characters of StimuliPresent and, after each rotation, calling Combinations on the tail of StimuliPresent. Table A1 shows the first two rotations of StimuliPresent that would occur in a call of GeneratePresentStimuliList (ABC). The number of rotations in each call of GeneratePresentStimuliList is equal to the length of the StimuliPresent string, and the last rotation returns StimuliPresent to its original state. The pseudocode runs:

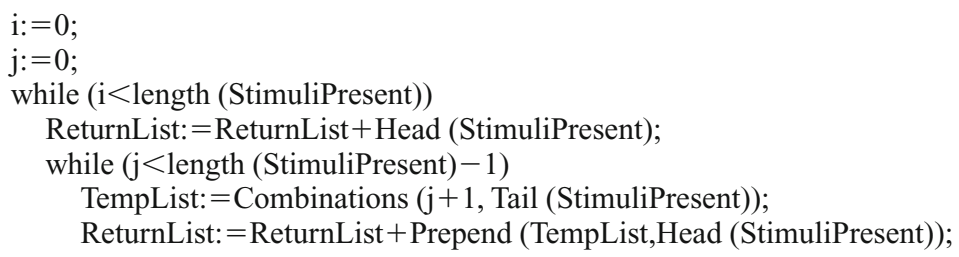




\begin{tabular}{c} 
\\
\hline $\mathrm{j}:=\mathrm{j}+1 ;$ \\
end while; \\
Rotate (StimuliPresent); \\
$\mathrm{i}:=\mathrm{i}+1 ;$ \\
end while;
\end{tabular}

Within this routine, the function Head returns the first character of its string parameter and the function Tail returns all characters of its parameter except the first. Rotate moves all characters of its parameter to the left, and the "overflow" character is appended to the end, as indicated in Table A1. Prepend is a function that takes a list of strings as a target, and for every string in that list, it prepends a character before returning the list with the prepends. The result of the call GeneratePresentStimuliList (ABC) returns a list consisting of the elements A, $\mathrm{AB}, \mathrm{AC}, \mathrm{ABC}$; $\mathrm{B}, \mathrm{BC}, \mathrm{BA}, \mathrm{BCA} ; \mathrm{C}, \mathrm{CA}, \mathrm{CB}, \mathrm{CAB}$. The elements listed after semicolons occur after rotations of StimuliPresent.

The final function of REMElements is Merge (AList,PList). It works by creating a list of strings that contains each element of PList plus each element of PList combined with each element of AList, as follows:

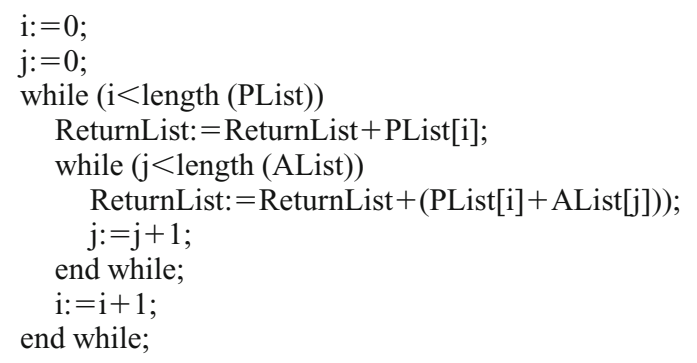

Using the examples above, if PList consisted of elements $\mathrm{A}, \mathrm{AB}, \mathrm{AC}, \mathrm{ABC}$; B, BC, BA, BCA; C, CA, $\mathrm{CB}, \mathrm{CAB}$, and $\mathrm{AList}$ consisted of elements $\mathrm{d}$, e, de, as they would after GenerateAbsentStimuliList (de) and GeneratePresentStimuliList (ABC), then Merge (AList, PList) would result in the list shown in Table 5. Thus, in a simulation of a five-component world (A-E), presentation of an $\mathrm{ABC}$ compound would result in activation of $16 \mathrm{~A}$ elements, $16 \mathrm{~B}$ elements, and $16 \mathrm{C}$ elements. This can be compared to simulation of the three-component world, where $\mathrm{ABC}$ would activate $4 \mathrm{~A}, 4 \mathrm{~B}$, and $4 \mathrm{C}$ elements. It should be noted, however, that the REM element weights that would be applied to each element ensure that the summed weights for an ABC stimulus always amount to 3, regardless of the "dimensionality" selected for the simulation (see text).

Table A1

Stimulus-Present Strings After Rotations in the

\begin{tabular}{ccc}
\multicolumn{3}{c}{$\begin{array}{c}\text { Function REMElements } \\
\text { A }\end{array}$} \\
B & C \\
B & C & A \\
C & A & B \\
\hline
\end{tabular}

Note-Boldface indicates the tails used in the calls on combinations used in the function GeneratePresentStimuliList.

(Manuscript received August 24, 2006;

revision accepted for publication October 13, 2006.) 